\title{
Etnografiando una disputa cultural: tensiones y sentidos en torno a la Educación Sexual Integral desde una perspectiva feminista
}

( Luciana Lavigne*

La convocatoria para tramar este debate colectivo ${ }^{1}$ en torno a la Educación Sexual Integral (ESI) se materializa en tiempos convulsionados en los que se tejen múltiples transformaciones: en legislaciones en pos del reconocimiento de derechos sexuales y reproductivos, en diversas formas de participación y construcción de demandas políticas y en la emergencia de nuevos sujetos políticos. Parto de considerar la dimensión política de la sexualidad, tal como mostró la antropóloga feminista Gayle Rubin: "el sexo es siempre algo político" (Rubin, 1989: 114). El interrogante que se habilita es: ¿qué sentidos, alcances y posibilidades de la política de ESI se producen en el escenario actual?

Para problematizar las tensiones y sentidos de la ESI retomo algunas reflexiones surgidas de un estudio etnográfico ${ }^{2}$ que realicé y de algunas escenas de mi labor como trabajadora de la educación en ámbitos de capacitación y formación docente en ESI en la Ciudad de Buenos Aires.

En primer lugar, quiero presentar brevemente un marco conceptual desde la antropología política para aproximarnos a este proceso de construcción social de la política de ESI. Esta perspectiva permite discutir el provocador interrogante acerca de si la ESI es o puede ser feminista. Considero que no hay una voz unívoca sobre lxs sujetos que encarnamos prácticas feministas y tensionamos sentidos al interior de las instituciones y en los ámbitos sociales donde la ESI cobra valor de disputa.

Los aportes de la antropología política permiten poner en perspectiva crítica y comprender los modos de funcionamiento de las políticas, en tanto símbolos, estatutos de legitimidad, tecnologías políticas, formas de gubernamentalidad e instrumentos de poder (Shore, 2010). Un enfoque antropológico de las políticas públicas implica revisar las premisas y sentidos que signan las definiciones de los problemas, las clasificaciones particulares de las poblaciones que son gobernadas y las legitimaciones de ciertos modos de intervención. Esta aproximación antropológica permite destacar su ambigüedad y polisemia, sus múltiples significados (Shore, 2010) y mostrar la coexistencia de diversas prácticas y sentidos asignados a la ESI por los actores sociales involucrados. Desde esta perspectiva entiendo entonces los heterogéneos posicionamientos en relación a la ESI, con matices y sentidos diversos, definida por los sujetos a partir de lo que dicen y hacen-con sus contradicciones y tensiones-. Implica identificar las maneras en que los sujetos confrontan, subvierten, manipulan o internalizan las definiciones contenidas en políticas públicas, es decir, explorar: ¿cuáles son las formas de recepción y los modos de experimentar las políticas públicas por quienes se ven afectadas por ellas en su vida cotidiana?, así como también reconocer los aportes de los sujetos en la construcción, activando demandas y disputas. Se trata
* Doctora en Antropología, Profesora y Licenciada en Ciencias Antropológicas, Facultad de Filosofía y Letras de la Universidad de Buenos Aires. Docente y capacitadora de educación sexual integral (ESI) en el Ministerio de Educación de la Ciudad de Buenos Aires. Ha participado como tallerista y docente de cursos virtuales del Programa Nacional de ESI. Ha desarrollado diversas investigaciones y publicaciones sobre cuerpos, géneros, sexualidades y educación. Integra diversos equipos de investigación en el IIEG y el ICA, FFyL-UBA. Y se desempeña como docente de la Carrera de Especialización en Educación: Pedagogías para la igualdad en contextos socioeducativos diversos, contextos socioeducativos diversos, dades; y de Teoría General del Movimiento, del Departamento de Artes.

1. Quiero agradecer a Alba Rueda por nuestras luchas compartidas y las conversaciones que nos dimos en la construcción de una narrativa posible para tramar este debate. También agradezco a Miranda González labor docente hace años, en pos de una educación pública ni sexista, ni heterosexista, ni androcéntrica y antirracista. Y a Alicia Comas por la sororidad permanente.

2. Tesis doctoral: Una etnografía sobre sexualidades, género y educación. La educación sexual integral en la Ciudad de Buenos Aires como política de gestión de la sexualidad juvenil. 2016. FFyL, UBA. 
de reconocer y problematizar estas diversas maneras de tramar, habitar y encarnar las políticas desde sujetos específicos e históricamente situados.

Fueron los movimientos sociales y sociosexuales de mujeres y feminismos quienes impulsaron el enfoque de la sexualidad desde la perspectiva de los derechos humanos y demandaron derechos sexuales, reproductivos y de género, que son hoy el sustrato para el abordaje de los contenidos visibles en la elaboración de las políticas públicas que atienden estas cuestiones. Desde la década de 1980, luego de la larga duración de las dictaduras latinoamericanas y con el retorno de la democracia, se profundizaron procesos de construcción de derechos. En nuestro país, durante los últimos años, un conjunto de políticas se hicieron eco de este proceso y se definieron políticas específicas de sexualidad.

En octubre de 2006 se definía una política pública educativa de orden nacional a partir de la sanción de la Ley 26150 que creó el Programa Nacional de Educación Sexual Integral y, en la Ciudad de Buenos Aires, se sancionaba la Ley de Educación Sexual Integral (Ley 2110/06). Se establecía así la ESI para toda la población escolarizada en los establecimientos educativos, en todos los niveles y modalidades del sistema público de gestión estatal y privada, y en las carreras de formación docente. El consenso expresado en los marcos normativos, como toda definición política, estuvo plagado de demandas, disputas y negociaciones. El proceso de construcción de la ESI en la Ciudad fue el resultado de diferentes intereses, proceso que significó una profunda disputa cultural. Se produjo una lucha política por los sentidos en torno a géneros, sexualidades, identidades y orientaciones afectivo-eróticas, en el marco de los debates y negociaciones para definir la sanción de la norma escrita, así como en cada ámbito donde los actores del campo encarnaron la política educativa.

Comprender la especificidad de este contexto histórico político implicó reconocer, en primer lugar, que el Estado - de un modo o de otro-interviene en la regulación de la sexualidad de la población. Así, retomé los enfoques que fundamentan que "las escuelas siempre educaron en sexualidad" y que sostienen que el dispositivo pedagógico escolar de modo intrínseco y constitutivo supone la regulación de las sexualidades y las identidades sexo-genéricas (Foucault, 2002 y Morgade y Alonso, 2008). Desde este punto de partida, entiendo que la política de educación sexual integral habilitó una manera específica de gestionar la sexualidad de la población escolarizada.

Aun cuando existieron innumerables desacuerdos entre los actores sociales que construyeron estas definiciones y estrategias de intervención, uno de los sentidos fundamentales consensuados en este proceso de construcción consistió en la denominación de integral. La integralidad de la Ley implicó una concepción de la sexualidad enfocada desde la diversidad de sus dimensiones, que incorpora aspectos físicos, emocionales, intelectuales y sociales; y a su vez considera el amor, la protección y el cuidado y el respeto a la diversidad de valores en sexualidad, entre otros. El propósito de esta definición fue modificar las concepciones de sexualidad que la restringían al sexo en términos biológicos, enfatizando la genitalidad y la reproducción como cuestiones centrales de la enseñanza (Elizalde, Felitti y Queirolo, 2009).

En el marco de su implementación, aunque el enfoque de la prevención siguió siendo en muchos casos dominante, otras perspectivas más centradas en los placeres, que en los riesgos y peligros asociados a la sexualidad, encontraron resonancias. Aquí podemos reconocer un derecho fundamental en la agenda del feminismo: el placer, tal como lo destacara Carol Vance (1989), haciendo tambalear la predominancia de los peligros por sobre los placeres. De alguna manera se produce un movimiento en las concepciones dominantes que ubicaron al placer sexual en el polo negativo 
del sistema de las jerarquías sexuales (Rubin, 1989), pero dependerá de las apuestas pedagógicas, políticas y epistemológicas de quienes encarnen la ESI.

Movimientos de mujeres, activistas y académicas feministas y de la disidencia sexual han reiterado las demandas de incorporación de una perspectiva de género en la formulación de las políticas, demandaron la necesidad imperiosa de reivindicar y visibilizar las diversidades sexuales, acabar con todas las formas de discriminación y contribuyeron con ideas fundamentales: la perspectiva de género, la deconstrucción del determinismo biológico, el cuestionamiento a la normativización de los procesos de socialización que construyen identidades y a los comportamientos estereotipados de la sexualidad, de lo femenino y lo masculino. La incorporación de la perspectiva de género en la ESI fue posible a partir de los principios básicos del modelo hermenéutico que elaboró el feminismo para explicar las desigualdades entre hombres y mujeres en términos sociales, culturales e históricos (Boccardi, 2008).

El enfoque de género, aunque ya formaba parte del campo discursivo en textos normativos desde la década de 1990, seguía generando disensos y tensiones para encuadrar sus alcances y significaciones. En esta perspectiva encontramos que la Ley de ESI de la Ciudad de Buenos Aires presenta un "umbral de tolerancia" (Cepeda, 2007) diferente con respecto a la ley nacional, en cuanto a la incorporación explícita de la categoría de género. Si bien la Ley porteña incorporó, finalmente, el enfoque de la perspectiva de género, la Iglesia y los sectores conservadores ofrecieron una dura resistencia y mantuvieron hasta último momento abierto el debate respecto de su incorporación.

Otra apuesta del feminismo implica la deconstrucción del lenguaje -en tanto institucionalizador de normas sociales- con el propósito de desnaturalizar y resignificar su carácter androcéntrico, sexista y heteronormativo. Se trata de una intervención política que en nuestro país fue realizada de muchas formas con el propósito de desarmar el dispositivo lingüístico que perpetúa relaciones sociales asimétricas. Encontramos que es incorporada, en algunos casos naturalizada, incluso institucionalizada, y cabe preguntarnos por los efectos de la estabilización de las significaciones disidentes con que fue promovida. Aun así, algunas apuestas se desencadenan y producen nuevas pedagogías, tales como agrietar el uso gramatical del género masculino como neutro de nuestra cultura falogocéntrica, crear otras expresividades que deconstruyan el sentido unívoco con marcaciones ( $x$, *, @, e, etcétera) en la lengua escrita, fundamentalmente -también hablada- , y recuperar las voces silenciadas, omitidas. ${ }^{3}$

3. En este escrito me apropio de una manera de decir, transitoria que introduce la marcación del género con la $\mathrm{x}$, a sabiendas que en otro contexto, otras formas expresivas podrían reemplazarla.

\section{Del conocimiento situado y las prácticas de concienciación}

Así como el feminismo reconoció la necesidad de reflexionar sobre el conocimiento situado (Haraway, 1991), a lo largo de los diversos cursos y capacitaciones docentes en las que he participado como capacitadora e incluso como observadora participante se reconocieron los posicionamientos subjetivos como una forma de producir conocimiento, en tanto determinan modos de actuar, de interpretar la vida, de configurar cosmovisiones y perspectivas, de nombrar las experiencias y de relacionarse con los demás. Este ejercicio de autoreflexión permitió reconocer que no solo la ESI involucra la subjetividad sino que en toda práctica docente siempre está implicado nuestro ser sexuado. Nuevamente, como formulara el feminismo, se muestra que la sexualidad ha sido colocada en el orden lo privado, pero, en realidad, es un tema del orden de lo público. Esta es la reconfiguración que aconteció a partir del abordaje de la ESI, que situó con estatuto "público" temas, enfoques y contenidos considerados del orden de "lo privado" (Tarzibachi, 2005). 
4. Que se encuentra en las puertas de entrada de los recursos elaborados por el Programa Nacional de ESI. Cuadernos de ESI, Ministerio de Educación de la Nación.
En las capacitaciones docentes se propusieron formas de producción de conocimiento que rescataran reflexiones teóricas a través de la primera persona del singular y la introspección, como un ejercicio político que transforma la experiencia personal en política. Reflexioné al respecto con mi pareja pedagógica (Lavigne y González Martin, 2015), con quien desplegamos espacios de formación docente en ESI hace años en la Ciudad de Buenos Aires, en la provincia de Buenos Aires y otras jurisdicciones. Coincidimos en reconocer que la política de ESI ha recuperado varias experiencias de la práctica feminista, a la manera de los grupos de concienciación, a partir de "la reflexión sobre nosotrxs mismxs", 4 sobre todo para propiciar la "sensibilización" docente y como estrategia que permita bucear, revelar, interpelar los supuestos y representaciones con que habitamos las escenas escolares -entre otras- cotidianamente. Implica habilitar las narraciones en primera persona para trasvasarlas al posible aprendizaje colectivo. Ingresaron entonces con gran relevancia las pasiones, los deseos, las emociones al territorio de la producción de conocimiento, y se incorporaron sentidos que redefinen la asepsia de los cuerpos sexuados en las escuelas para reposicionarlos en su integralidad.

\section{Acerca de los procesos de politización}

En cuanto a las diversas maneras de tramar la ESI reconocemos aquí formas de confrontarla, subvertirla y traccionarla por parte de diversos actores que complejizan las orientaciones iniciales desde procesos de organización y demanda colectiva con incidencia en las configuraciones de esta política pública.

Para muchxs actores del campo educativo, la política de ESI implicó una militancia, que fue definida reiteradamente en términos de "ponerse la camiseta" [de la ESI] en un doble reconocimiento: por un lado, promover los derechos de niñxs y jóvenes a recibir educación e información sobre sexualidades, y a su vez, por otro lado, en defensa del derecho de lxs propixs docentes a ser habilitadxs para desarrollar estos temas. La concepción de la docencia y algunas prácticas asociadas al proceso de emancipación, de promoción del ejercicio de los derechos, como los derechos sexuales y reproductivos, han quedado asociados en el imaginario social como prácticas asimilables a la experiencia de la militancia. Esta concepción militante supone un proceso de politización que de alguna forma socava la mera significación de la tarea docente como un trabajo en el campo de la educación para agregar un sentido de compromiso, convicciones y experiencias activistas entre las personas que han asumido y encarnado la ESI.

Pero también el estudiantado emerge en este contexto como actor político central que contribuye en esta arena de disputas por los sentidos. Actualmente, las juventudes no solo son interlocutores significativos, sino que también son ineludibles para comprender el proceso de construcción de esta política. Mientras que la política pública se ha caracterizado por ser adultocéntrica, y su construcción inicial conllevó la ausencia de la voz de lxs jóvenes (Lavigne, 2011), pensadxs como población destinataria más que como actores sociales que construyen disputas de sentidos en la escena política, la ESI se tornó en protagonista como un campo de posibilidades, como estrategia discursiva, incluso como praxis feminista, en la propia disputa por las demandas y reivindicaciones de deconstrucción de formas jerárquicas del saber escolar al reclamar por sus derechos e interpelando a lxs adultxs para abordar temas silenciados, incómodos. La defensa por la educación pública del estudiantado fue atravesada por consignas que involucraron con profundidad política una concepción de la sexualidad que produjo una apropiación de sentidos que el sexo siempre es algo político. Esto implicó que jóvenes enarbolaron consignas históricas del feminismo, en 
las marchas, en los centros de estudiantes, en las tomas de las escuelas secundarias y diversos contextos públicos donde mostraron que la ESI era apropiada como una oportunidad para interpelar la cultura cisheteropatriarcal. Demandaron la efectiva implementación de la ESI y la urgente creación de protocolos de prevención y erradicación de la violencia de género y la discriminación. ${ }^{5}$ Así devinieron en sujetos activos de la arena de disputas que contribuyeron a resquebrajar sentidos hegemónicos, haciendo colapsar el lenguaje, visibilizando identidades sexo-genéricas que escapan a la norma y denunciando la reproducción de las violencias de género. Comenzaron a proliferar "comisiones de género" en las escuelas secundarias como espacios privilegiados para reflexionar e interpelar las formas en que se perpetúan las desigualdades sociales, políticas y económicas, y lo hicieron asumiendo una perspectiva de género porque encontraron que les permitía problematizar y comprender las relaciones de poder y las formas de dominación y subordinación históricas.

Otros territorios políticos de articulación encontramos cuando reconocemos que el proceso de construcción social de la ESI se enlaza con procesos hoy globales, como la masiva convocatoria promovida por la colectiva feminista Ni una menos. En este contexto, el impacto de estas movilizaciones sociales y sus consignas, como "Sin ESI no hay ni una menos", significaron la visibilización de problemáticas sociales absolutamente pertinentes para quienes encarnamos abordajes de la ESI. La violencia de género comenzó a ser una experiencia visibilizada que solapaba estas "situaciones problemáticas" a una misma vez en el campo social general y en particular en los ámbitos educativos. Las demandas históricas colectivas de los movimientos sociosexuales para acabar con esta forma específica de violencia se canalizaron en una política de estado que, haciéndose eco de la Ley Nacional 26485/09 "Protección integral para prevenir, sancionar y erradicar la violencia contra las mujeres en los ámbitos en los que desarrollen sus relaciones interpersonales", creó otra Ley profundamente vinculada para estimular este abordaje en las escuelas: Ley Nacional 27234/15, que dispone en la agenda educativa jornadas institucionales en las escuelas de todos los niveles educativos denominadas: "Educar en Igualdad, Prevención y Erradicación de la Violencia de Género". Una vez más, abierto el espacio escolar, las demandas por la construcción de herramientas como protocolos, espacios para problematizar, denunciar y acompañar a jóvenes que experimentaran estas situaciones, comenzaron a ser necesidades que surcaron las relaciones juveniles, entre generaciones e intra-generacionales.

Estas trayectorias políticas requieren de una genealogía que permita contextualizar y reconocer procesos históricos locales. Con el fervor de la recuperación democrática en la década de 1980, se produce una particular movilización colectiva de mujeres que de modo ininterrumpido habitaron encuentros anuales denominados Encuentros Nacionales de Mujeres como semillero de praxis feministas, pero también de mujeres organizadas en barrios populares cuyas trayectorias se fueron enriqueciendo recíprocamente, que en los últimos años devinieron masivos. Se entroncan de particular manera con la ESI y desde hace unos años se incluyó un taller específico denominado: "Estrategias para la implementación de la ESI", que expone la necesidad de debatir y tramar metodologías para garantizar su abordaje.

Mientras producimos este debate, la ESI es traccionada por una demanda histórica del movimiento feminista: el derecho al aborto. La interrupción legal del embarazo impulsada por la "Campaña Nacional por el Derecho al Aborto Legal Seguro y Gratuito" confluyó en la estrategia de construcción política parlamentaria a partir de la formulación de proyectos para devenir en una ley, con la toma de las calles por las juventudes enfervorizadas e históricas militantes en una ya instalada metáfora para describir las multitudes movilizadas: "marea verde". ${ }^{6}$ Se trata de un contenido curricular de la ESI incluido entre los lineamientos nacionales ${ }^{7}$ y en el diseño curricular
5. Recientemente fue creado un protocolo para ser aplicado en los establecimientos educativos de la Ciudad de Buenos Aires. Lo interesante es destacar que en la comunicación oficial realizada desde la esfera del

Ministerio de Educación e Innovación, se reconoce explícitamente que "fue elaborado a partir de las demandas e inquietudes de los/as estudiantes [...] El mismo llega en un momento histórico en el cual la conciencia profunda de los adolescentes en profunda de los adolescentes en temas de género nos interpela y nos exige estar a la altura". Resta
indagar las formas en que se aplicará este instrumento en las prácticas institucionales cotidianas para que constituya un herramienta que permita tanto prevenir como intervenir ante estas situaciones. "PROTOCOLO DE ACCIÓN INSTITUCIONAL DE ACCIÓN INSTITUCIONAL
EN ESCUELAS SECUNDARIAS Y EN ESCUELAS SECUNDARIAS Y
ESTABLECIMIENTOS TERCIARIOS PARA LA PREVENCIÓN E INTERVENCIÓN ANTE SITUACIONES DE VIOLENCIA DE GÉNERO Y DISCRIMINACIÓN BASADA EN LA ORIENTACIÓN SEXUAL E IDENTIDAD DE GÉNERO O SU EXPRESIÓN", Junio, 2018.
6. En referencia al pañuelo usado como emblema de la lucha por la despenalización y legalización del aborto por la Campaña.

7. Lineamientos curriculares para la educación sexual integral. Ministerio de Educación de la Nación, 2008. 
8. Diseño Curricular para la Nueva Escuela Secundaria de la Ciudad de Buenos Aires. Ministerio de Educación. 2015.

9. Ley 26754/12 Establécese el derecho a la identidad de género de las personas.

10. Ley $26618 / 10$ de Matrimonio Civil. de ESI en la NES, ${ }^{8}$ pero que viene siendo abordado con muchas dificultades, resistencias y desafíos. Un marco normativo que regule estas prácticas como una política pública que garantice el acceso seguro y gratuito reconvierte lo decible y reformula las intervenciones políticas también en el campo educativo. De hecho, recordemos la consigna central con que se militó la Ley, "Educación sexual para decidir, anticonceptivos para no abortar, aborto legal para no morir", que involucró la ESI entendida como una estrategia pedagógica para promover herramientas para la toma de decisiones sobre el propio cuerpo.

Ahora bien, la ESI se encuentra permeada y resignificada permanentemente a raíz de la construcción de otras políticas, producto de demandas también colectivas y aunque resulta imposible incluir un detalle de la cronología de estas orientaciones, resulta significativo mencionar dos leyes que resignificaron los alcances de la ESI: la Ley de Identidad de género' y de Matrimonio Igualitario ${ }^{10}$ que impulsaron otros sentidos de los enfoques de la ESI.

\section{Para seguir tramando ESI(s)...}

Estas experiencias y la vigencia de las leyes ESI hace ya más de una década, nos llevan a preguntarnos por ¿cuáles son los horizontes de subjetivación para niñxs, jóvenes y adultxs en las escuelas?, ¿cuáles son las trayectorias escolares disímiles que podrán desplegarse?, ¿cómo se construyen las transformaciones en las tramas del saber y la política de conocimiento trazadas en las escuelas? La expectativa que adelanto es la reterritorialización de la formulación de las preguntas plausibles, de la ignorancia y la curiosidad (Britzman, 2001) habilitadas dentro del espacio escolar.

Sostengo que si bien la ESI es una técnica de poder, de control, regulación y de gestión de la sexualidad, al mismo tiempo, representa una oportunidad de desarrollar un paradigma de conocimiento respecto de las sexualidades, que permite interpelar, agrietar y/o desarticular concepciones dominantes basadas en la heteronormatividad, promover saberes impulsados por académicxs y los movimientos feministas/de mujeres y sociosexuales y la producción de otras epistemologías -como las feministas-en las instituciones educativas. En tanto que las pedagogías involucran intereses políticos, no son ni neutras, ni imparciales, ni universales, ni objetivas, en ellas se despliegan contradicciones, disputas culturales e ideológicas particulares. Los modos de encarnar la política de ESI, los modos de significarla y de desplegar las prácticas pedagógicas en relación con la sexualidad de niñxs, jóvenes y adultxs deben entenderse desde una diversidad de posiciones y contextos.

El desafío de reflexionar en torno a la ESI multiplicó los interrogantes desde un puerperio reciente, pero con la certeza que el feminismo nos ha dado algunas herramientas para producir conocimiento desde nuestro ser en el mundo multisituado que activa una praxis feminista que reconoce las experiencias personales como materia prima para la producción de saberes y conocimientos. En este contexto, muchxs encarnamos pedagogías feministas como modo de asumir la lucha por eliminar toda forma de discriminación, de explotación o de opresión (clase, raza, sexo, etnia, orientación sexual, edad, etcétera). Y esta apuesta queda resonando como un desafío para mi compromiso político con la educación pública, como antropóloga, feminista, madre, mujer y docente. 


\section{Dibliografía}

» Boccardi, F. (s/f). "Los efectos del feminismo y los estudios de género en la educación sexual”. AlFilo. Recuperado de https://ffyh.unc.edu.ar/alfilo/anteriores/24/boccardi-los-efectos-del-feminismo-y-los-estudios-de-genero.pdf

» Britzman, D. (2001). “Curiosidade, sexualidade e curriculo". En G. Louro, O corpo educado. Pedagogías da sexualidade (pp. 83-111). Belo Horizonte, Brasil: Autêntica.

"Cepeda, A. (2007). “De eso sí habla. Los debates de la Ley de Educación sexual en Argentina". II Coloquio Interdisciplinario Internacional. Educación, sexualidades y relaciones de género. Investigaciones y experiencias. Facultad de Filosofía y Letras. Universidad de Buenos Aires. 1, 2 y 3 de agosto.

»Elizalde, S. Felitti, K. y Quierolo, G. (Coords). (2009). Género y sexualidades en las tramas del saber. Revisiones y propuestas. Buenos Aires, Argentina: Libros del Zorzal.

"Foucault, M. (2002). Historia de la Sexualidad. La voluntad de saber. Tomo I. Buenos Aires: Siglo veintiuno editores.

" Haraway, D. (1991). Ciencia, cyborgs y mujeres. La reinvención de la naturaleza. Madrid, España: Cátedra.

»Lavigne, L. y Gonzalez Martin, M. (2015). "Reflexiones desde la formación docente en educación sexual integral. Una propuesta para el abordaje de la diversidad sexual humana". Revista Temas de Educación, 21/1, 183-200.

" Lavigne, L. (2011). “Las sexualidades juveniles en la educación sexual integral”. En S. Elizalde (Coord.), Jóvenes en cuestión. Configuraciones de género y sexualidad en la cultura (pp. 87-118). Buenos Aires, Argentina: Biblos.

» Morgade, G. y Alonso, G., (2008). Cuerpos y sexualidades en la escuela. De la "normalidad" a la disidencia. Buenos Aires, Argentina: Paidós.

» Rubin, G. (1989). “Reflexionando sobre el sexo: notas para una teoría radical de la sexualidad". En C. S. Vance (comp.), Placer y peligro. Explorando la sexualidad femenina (pp. 113-190). Madrid, España: Revolución.

»Shore, C. (2010). “La antropología y el estudio de la política pública: reflexiones sobre la ‘formulación' de las políticas”. Antípoda. Revista de Antropología y Arqueología, 10, 21-49.

"Tarzibachi, E. (2005). “La Educación Sexual en el intersticio entre lo público y lo privado". Revista Novedades Educativas, 178, 62-65.

"Vance, C. (1991). Placer y Peligro. Explorando la sexualidad femenina. Madrid, España: Revolución. 
